\title{
Impulsive Synchronization of the Neural Networks with a Gui Chaotic Strange Attractor
}

\author{
Kaihua Wang ${ }^{1}$ and Zhanji Gui ${ }^{2, *}$ \\ ${ }^{1}$ School of Mathematics and Statistics, Hainan Normal University, Haikou, Hainan, P.R. China \\ ${ }^{2}$ Department of Software Engineering, Hainan College of Software Technology, Qionghai, P.R. China \\ ${ }^{*}$ Corresponding author
}

\begin{abstract}
Gui chaotic strange attractor is studied. By employing the Lyapunovlike stability theory of impulsive functional differential equations, some criteria for synchronization between two impulsive neural networks are derived. An illustrative example is provided to show the effectiveness and feasibility of the proposed method and results.
\end{abstract}

Keywords-neural networks; Gui chaotic strange attractor; synchronization

\section{INTRODUCTION}

Neural networks are attached more importance by many researchers, due to their widely applications in signal processing, pattern recognition, optimization, and so on ([1][4]). On the other hand, in real world, many evolutionary processes are characterized by abrupt changes at certain time. These changes are called to be impulsive phenomena, which are included in many fields such as physics, chemistry, population dynamics, optimal control, etc. and can be described by impulsive differential equations (see [5]). Thus, researches of neural networks with impulse have been received much interesting ([6]-[8]). In recent years, the additive neural networks have been extensively studied, including both continuous time and discrete-time settings, and applied to associative memory, model identification, optimization problems, etc. Many essential features of these networks, such as qualitative properties of stability, oscillation, \& convergence issues have been widely investigated ([9]-[12]).

However, as we all know, in many applications, the property of periodic oscillatory solutions or chaotic attractor of cellular neural networks is of great interest. In fact, there has been considerable research on the nonautonomous neural networks, such as [9], [13]. Meanwhile, the cellular neural networks with impulse effect are also widely studied, where the criteria on the existence, uniqueness and global stability of periodic solution or chaotic attractor are obtained. Further, a new chaotic strange attractor was also found, known as Gui chaotic strange attractor (eg. [14]-[18]).

Another type of synchronization, impulsive synchronization, has been developed (see [19]). It allows synchronization of chaotic systems using only small impulses generated by samples of the state variables of the driving system at discrete time instances [20]. These samples are called the synchronizing impulses and they drive the response system discretely at these instances. After a finite period of time, the two chaotic systems behave in accordance with each other and the synchronization of the two chaotic systems is achieved. In other words, the asymptotic stability property of the error dynamics between the driving and response systems is reached. The impulsive synchronization has been applied to a number of chaotic based communication systems which exhibit good performance for synchronization purposes and for security purposes ([21], [22]).

Motivated by the above discussions, the aim of this paper is to study the synchronization of impulsive neural networks with a Gui chaotic strange attractor. By employing the Lyapunovlike stability theory of impulsive functional differential equations, some criteria for synchronization of impulsive neural networks are derived.

The remainder of this paper is organized as follows: Section II describes the issue of synchronization of coupled impulsive systems with a Gui chaotic strange attractor. In Section III, some sufficient conditions for the synchronization are derived by constructing suitable Lyapunov-like function. In Section IV, an illustrative example is given to show the effectiveness of the proposed method. Finally, conclusions are given in Section V.

\section{PRELIMINARIES AND PROBLEM FORMULATION}

In this paper, we consider the following cellular neural networks model with impulses

$$
\left\{\begin{array}{l}
\frac{d x_{i}(t)}{d t}=-a_{i} x_{i}(t)+\sum_{j=1}^{n} b_{i j} f_{j}\left(x_{j}(t)\right), \quad t \neq t_{k} \\
\Delta x_{i}\left(t_{k}\right)=x_{i}\left(t_{k}^{+}\right)-x_{i}\left(t_{k}^{-}\right)=d_{i k} x_{i}\left(t_{k}^{-}\right)
\end{array}\right.
$$

where $i=1,2, \ldots, n ; k=1,2, \ldots, \Delta x_{i}\left(t_{k}\right)=x_{i}\left(t_{k}^{+}\right)-x_{i}\left(t_{k}^{-}\right)$are the impulses at moments $t_{k}$ and $t_{1}<t_{2}<\cdots$ is a strictly increasing sequence such that $\lim _{k \rightarrow \infty} t_{k}=+\infty ; x_{i}(t)$ corresponds to the state of the $i$ th unit at time $t, f_{j}\left(x_{j}(t)\right)$ denotes the output of the $j$ th unit at time $t, b_{i j}$ denotes the strength of the $j$ th unit on the $i$ th unit at time $t$ which satisfies $b_{i j}=0$ when $|i-j|>1, a_{i}$ represents the rate with which the $i$ th unit will reset its potential to the resting state when disconnected from the network and external inputs.

Throughout this paper, we assume that: 
$\left(\mathrm{H}_{1}\right)$ Functions $f_{j}(u) \quad(j=1,2, \ldots, n)$ are Lipschitz continuous and monotonically non-decreasing, i.e. for all $u_{1}, u_{2} \in \square=(-\infty, \infty)$ there are constants $L_{j}>0$ such that

$$
0 \leq \frac{f_{j}\left(u_{1}\right)-f_{j}\left(u_{2}\right)}{u_{1}-u_{2}} \leq L_{j} .
$$

$\left(\mathrm{H}_{2}\right)$ There exists a positive integer $T$, such that

$$
t_{k+T}=t_{k}+\omega, \quad d_{i(k+T)}=d_{i k},
$$

where $k=1,2, \ldots, i=1,2, \ldots, n$.

Now we consider the derive system in the form of the neural networks (1). For the purpose of synchronization, we introduce the response system driven by (1) via a set of signals

$$
\left\{\begin{array}{l}
\frac{d y_{i}(t)}{d t}=-a_{i} y_{i}(t)+\sum_{j=1}^{n} b_{i j} f_{j}\left(y_{j}(t)\right), \quad t \neq t_{k} \\
y_{i}\left(t_{k}^{+}\right)=2 d_{i k} x_{i}\left(t_{k}^{-}\right)+\left(1-d_{i k}\right) y_{i}\left(t_{k}^{-}\right),
\end{array}\right.
$$

where $b_{i j}=0$ (when $|i-j|>1$ ), $i=1,2, \ldots, n, k=1,2, \ldots$.

Let $e(t)=y_{i}(t)-x_{i}(t)$ be synchronization error, where $e(t)=\left(e_{1}(t), e_{2}(t), \ldots, e_{n}(t)\right)^{T}, x_{i}(t)$ and $y_{i}(t)$ are the state variables of drive system (1) and response system (3). Thus, we can derive the error dynamical system as follows:

$$
\left\{\begin{array}{l}
\dot{e}=-D e(t)+W G(e(t)), \quad t \neq t_{k}, \\
e\left(t_{k}^{+}\right)=\left(I-D_{k}\right) e\left(t_{k}^{-}\right), \quad k=1,2, \ldots,
\end{array}\right.
$$

where $g_{j}=f_{j}\left(e_{j}+x_{j}\right)-f_{j}\left(x_{j}\right), G(e)=\left[g_{1}, g_{2}, \ldots, g_{n}\right]^{T}$.

$I$ is identity matrix, and $D=\left(b_{i j}\right)$, when $|i-j|>1, b_{i j}=0$.

$$
W=\left(\begin{array}{cccc}
-a_{1} & 0 & \cdots & 0 \\
0 & -a_{2} & \cdots & 0 \\
\vdots & \vdots & \ddots & \vdots \\
0 & 0 & \cdots & -a_{n}
\end{array}\right), D_{k}=\left(\begin{array}{cccc}
d_{1 k} & 0 & \cdots & 0 \\
0 & d_{2 k} & \cdots & 0 \\
\vdots & \vdots & \ddots & \vdots \\
0 & 0 & \cdots & d_{n k}
\end{array}\right)
$$

In fact, from the analysis above, we can see that (1) and (3) are synchronized if and only if the equilibrium point of (4) is asymptotically stable for any initial condition. So the global impulsive synchronization problem can be solved if the controller gain matrices $D_{k}$ are suitably designed such that the zero solution of (4) is globally asymptotically stable.

\section{MAIN RESULTS}

In this section, we will derive some sufficient conditions for synchronization between drive system (1) and response system (3) with impulsively controlling.

Theorem 1. If there exist a positive constant $\varepsilon>0, \alpha>0$, positive definite diagonal matrix $P>0$, such that

(1) Linear matrix inequality

$$
\left(\begin{array}{cc}
-P D-D^{T} P+\varepsilon L_{M}^{2} \lambda_{M}\left(W^{T} W\right)-\alpha P & P \\
P & -\varepsilon
\end{array}\right)<0
$$

where $L_{M}=\max \left\{L_{j}\right\}, \lambda_{M}\left(^{*}\right)$ denote the largest eigenvalue of matrix *;

(2) $\tau<\inf _{k \in \square}\left\{t_{k}-t_{k-1}\right\}<1$;

(3) There exists a constant $\eta>1$ such that

$$
\ln \left(\eta \beta_{k}\right)+\alpha\left(t_{k}-t_{k-1}\right)<0,
$$

where $\beta_{k}=\lambda_{M}^{2}\left(I-D_{k}\right)$;

then, the origin of system (4) is globally asymptotically stable, which implies that (1) and (3) are completely synchronized.

Proof: Construct a Lyapunov function in the form of

$$
V(e(t))=e^{T}(t) P e(t) .
$$

when $t \in\left(t_{k-1}, t_{k}\right]$, the total derivative of $V(e)$ with respect to (4) is

$$
\begin{aligned}
\dot{V}(e) & =\dot{e}^{T} P e+e^{T} P \dot{e} \\
& =(-D e+W G(e))^{T} P e+e^{T} P(-D e+W G(e)) \\
& =-e^{T}\left(P D+D^{T} P\right) e+2 e^{T} P W G(e) .
\end{aligned}
$$

Also, by the well-known inequality $2 a^{T} b \leq \varepsilon^{-1} a^{T} a+\varepsilon b^{T} b$, for $\forall \varepsilon>0$, we obtain

$$
\dot{V}(e) \leq-e^{T}\left(P D+D^{T} P\right) e+\varepsilon^{-1} e^{T} P P e+\varepsilon L_{M}^{2} \lambda_{M}\left(W^{T} W\right) G(e) .
$$

From Assumption (H1), we can obtain $\|G(e)\|^{2} \leq L_{M}^{2}\|e\|^{2}$, this leads to

$$
\dot{V}(e) \leq-e^{T}\left(P D+D^{T} P\right) e+\varepsilon^{-1} e^{T} P P e+\varepsilon L_{M}^{2} \lambda_{M}\left(W^{T} W\right) e^{T} e .
$$

From Linear matrix inequality (5) and (7), we have

$$
\dot{V}(e)<\alpha e^{T} P e=\alpha V(e) .
$$

Let $V(t)=V(e(t))$, then 


$$
V(t) \leq V\left(t_{k-1}^{+}\right) \exp \left[\alpha\left(t-t_{k-1}\right)\right],
$$

when ${ }^{t \in\left(t_{k-1}, t_{k}\right]}$, from the second equation in (4), we have

$$
\begin{aligned}
V\left(t_{k}^{+}\right) & =e^{T}\left(t_{k}^{+}\right) P e\left(t_{k}^{+}\right) \\
& =\left[\left(I-D_{k}\right) e\left(t_{k}\right)\right]^{T} P\left[\left(I-D_{k}\right) e\left(t_{k}\right)\right] \\
& \leq \lambda_{M}^{2}\left(I-D_{k}\right) V\left(t_{k}\right)=\beta_{k} V\left(t_{k}\right)
\end{aligned}
$$

For $t \in\left(t_{0}, t_{1}\right]$, from (8) and (9), we have

$$
V(t) \leq V\left(t_{0}\right) \exp \left[\alpha\left(t-t_{0}\right)\right], \quad t \in\left(t_{0}, t_{1}\right]
$$

which leads to

$$
\begin{gathered}
V\left(t_{1}\right) \leq V\left(t_{0}\right) \exp \left[\alpha\left(t_{1}-t_{0}\right)\right], \quad t \in\left(t_{0}, t_{1}\right], \\
V\left(t_{1}^{+}\right) \leq V\left(t_{0}\right) \beta_{1} \exp \left[\alpha\left(t_{1}-t_{0}\right)\right], \quad t \in\left(t_{0}, t_{1}\right] .
\end{gathered}
$$

Similarly, for $t \in\left(t_{1}, t_{2}\right]$,

$$
\begin{aligned}
V(t) & \leq V\left(t_{1}^{+}\right) \exp \left[\alpha\left(t-t_{1}\right)\right], \\
& \leq V\left(t_{0}\right) \beta_{1} \beta_{2} \exp \left[\alpha\left(t-t_{0}\right)\right] .
\end{aligned}
$$

In general, for $t \in\left(t_{k-1}, t_{k}\right]$,

$$
\begin{aligned}
V(t) & \leq V\left(t_{1}^{+}\right) \exp \left[\alpha\left(t-t_{1}\right)\right], \\
& \leq V\left(t_{0}\right) \beta_{1} \beta_{2} \cdots \beta_{k} \exp \left[\alpha\left(t-t_{0}\right)\right] .
\end{aligned}
$$

For $t \in\left(t_{k}, t_{k+1}\right]$, it follows from (6) and (10) that,

$$
\begin{aligned}
V(e(t)) \leq & V\left(e\left(t_{0}\right)\right) \beta_{1} \beta_{2} \cdots \beta_{k} \exp \left[\alpha\left(t-t_{0}\right)\right] \\
\leq & V\left(e\left(t_{0}\right)\right)\left\{\beta_{1} \exp \left[\alpha \tau_{1}\right]\right\}\left\{\beta_{2} \exp \left[\alpha \tau_{2}\right]\right\} \\
& \cdots\left\{\beta_{k} \exp \left[\alpha \tau_{k}\right]\right\} \exp \left[\alpha\left(t-t_{k}\right)\right] \\
\leq V\left(e\left(t_{0}\right)\right) & \frac{\exp \left[\alpha\left(t-t_{k}\right)\right]}{\eta^{k}}
\end{aligned}
$$

From (11), we can see that the trivial solution of system (4) is globally asymptotically stable. This completes the proof.

\section{AN ILLUSTRATIVE EXAMPLE}

In order to demonstrate and verify the performance of the proposed method, some numerical simulations are presented in this section.

As is known to all that (1) can exhibit Gui chaotic strange attractor. In order to show it clearly, we give the following example:

$$
\left\{\begin{array}{l}
\left(\begin{array}{l}
\dot{x}_{1} \\
\dot{x}_{2} \\
\dot{x}_{3}
\end{array}\right)=-\left(\begin{array}{l}
x_{1} \\
x_{2} \\
x_{3}
\end{array}\right)+\left(\begin{array}{ccc}
1.2 & -1.6 & 0 \\
1.2 & 1 & 0.9 \\
0 & 2.2 & 0.15
\end{array}\right)\left(\begin{array}{l}
f_{1}\left(x_{1}\right) \\
f_{2}\left(x_{2}\right) \\
f_{3}\left(x_{3}\right)
\end{array}\right) \\
x_{i}\left(t_{k}^{+}\right)=x_{i}\left(t_{k}^{-}\right)+d_{i k} x_{i}\left(t_{k}\right), \quad i=1,2,3, \quad k \in \square^{+}
\end{array}\right.
$$

where $d_{1 k}=0.35, \quad d_{2 k}=0.4, \quad d_{3 k}=0.5$,

$$
f_{j}\left(x_{j}\right)=\frac{1-e^{-2.5 x_{j}}}{1+e^{-2.5 x_{j}}}, \quad j=1,2,3 .
$$

Obviously, $f_{j}(x)$ satisfy (H1).

Numerical simulation indicate that system (12) without impulsive effect has a chaotic attractor (see Fig 1).

Now we investigate the influence of the period $T$ of impulsive effect on the system (12). Set $T=1$, then the original chaotic attractor of system (12) without impulsive effect will be destroyed by impulses. However, numeric results show that system (12) still has a global attractor which can be a Gui chaotic strange attractor (see Figs. 2-5).

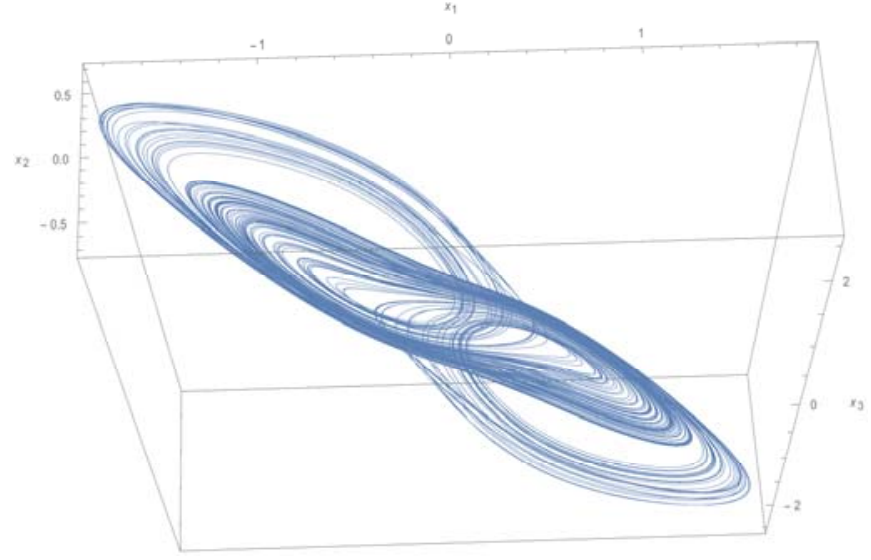

FIGURE I. CHAOTIC STRANGE ATTRACTOR OF SYSTEM (12) WITHOUT IMPULSIVE EFFECT

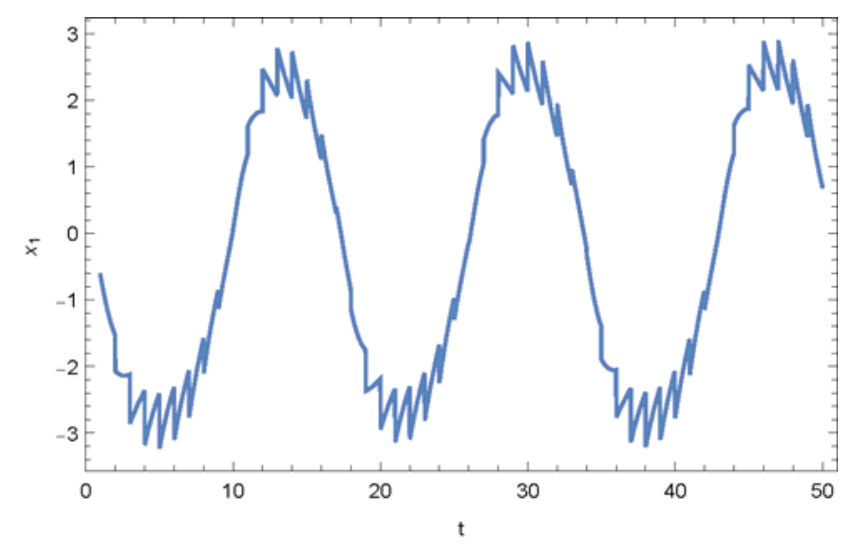

FIGURE II. TIME-SERIES OF $x_{1}(t)$ OF SYSTEM (12) WITH IMPULSE PERIOD $T=1$ 


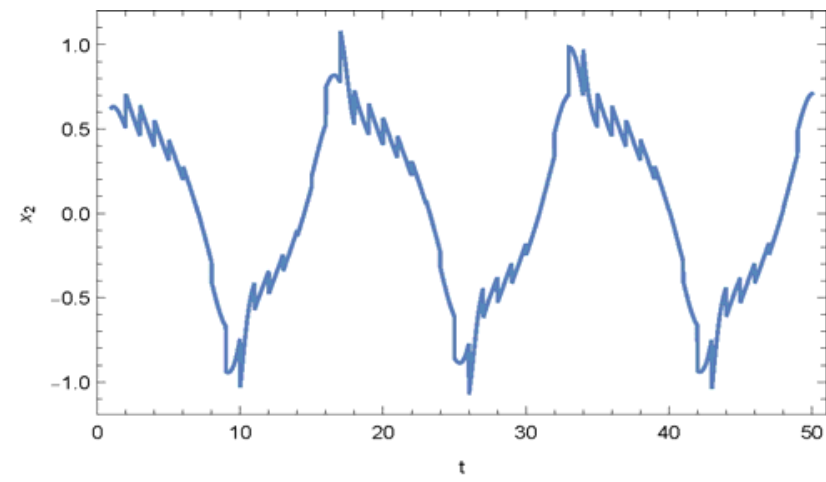

FIGURE III. TIME-SERIES OF $x_{2}(t)$ OF SYSTEM (12) WITH IMPULSE PERIOD $T=1$

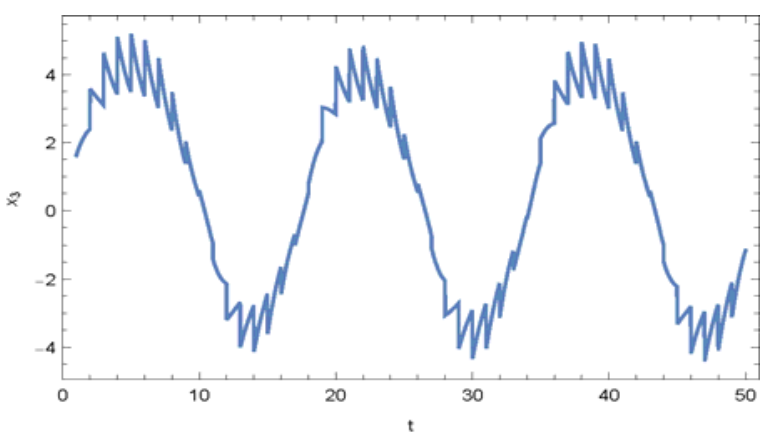

FIGURE IV. TIME-SERIES OF $x_{3}(t)$ OF SYSTEM (12) WITH IMPULSE PERIOD $T=1$

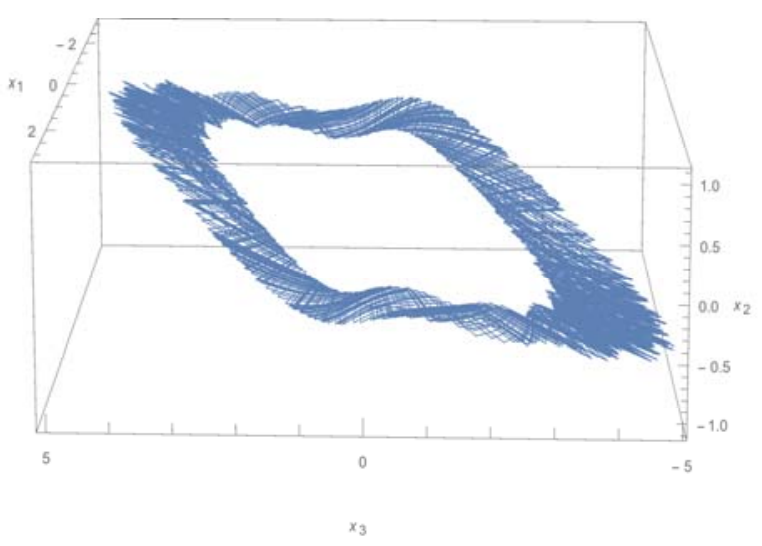

FIGURE V. PHASE PORTRAIT OF GUI CHAOTIC STRANGE =ATTRACTOR OF SYSTEM (12) WITH IMPULSE PERIOD $T=1$

Now the response chaotic cellular neural network is designed as follows:

$$
\left\{\begin{array}{l}
\left(\begin{array}{l}
\dot{y}_{1} \\
\dot{y}_{2} \\
\dot{y}_{3}
\end{array}\right)=-\left(\begin{array}{l}
y_{1} \\
y_{2} \\
y_{3}
\end{array}\right)+\left(\begin{array}{ccc}
1.2 & -1.6 & 0 \\
1.2 & 1 & 0.9 \\
0 & 2.2 & 0.15
\end{array}\right)\left(\begin{array}{l}
f_{1}\left(y_{1}\right) \\
f_{2}\left(y_{2}\right) \\
f_{3}\left(y_{3}\right)
\end{array}\right) \\
y_{i}\left(t_{k}^{+}\right)=2 d_{i k} x_{i}\left(t_{k}^{-}\right)+\left(1-d_{i k}\right) y_{i}\left(t_{k}^{-}\right), \quad i=1,2,3, \quad k \in \square^{+}
\end{array}\right.
$$

Let $e(t)=y_{i}(t)-x_{i}(t)$, then the error system (14) of drive system (12) and response system (13) is constructed as follow

$$
\left\{\begin{array}{l}
\left(\begin{array}{l}
\dot{e}_{1} \\
\dot{e}_{2} \\
\dot{e}_{3}
\end{array}\right)=-\left(\begin{array}{l}
e_{1} \\
e_{2} \\
e_{3}
\end{array}\right)+\left(\begin{array}{ccc}
1.2 & -1.6 & 0 \\
1.2 & 1 & 0.9 \\
0 & 2.2 & 0.15
\end{array}\right)\left(\begin{array}{c}
f_{1}\left(e_{1}+x_{1}\right)-f_{1}\left(x_{1}\right) \\
f_{2}\left(e_{2}+x_{2}\right)-f_{2}\left(x_{2}\right) \\
f_{3}\left(e_{3}+x_{3}\right)-f_{3}\left(x_{3}\right)
\end{array}\right) \\
e_{i}\left(t_{k}^{+}\right)=\left(1-d_{i k}\right) e_{i}\left(t_{k}^{-}\right), \quad i=1,2,3
\end{array}\right.
$$

If one choose $L_{1}=L_{2}=L_{3}=1.5, \eta=1.1, d_{1 k}=0.35$, $d_{2 k}=0.4, d_{3 k}=0.5$, it is easy to check the conditions in Theorem 1 are satisfied. So, the system (12) and (13) is synchronized. By Theorem 1, synchronization can be obtained. The synchronization performance is illustrated by Fig. 6, 7. The numerical simulations show that synchronization could be quickly achieved.

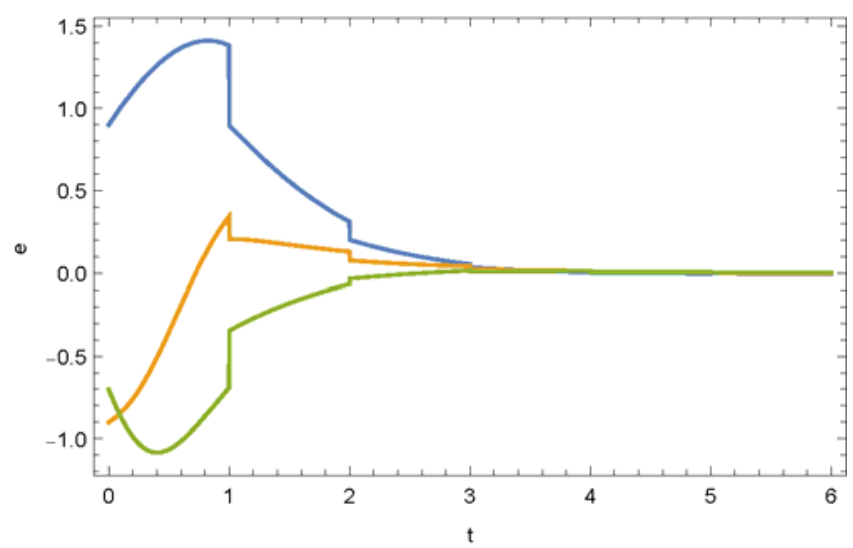

FIGURE VI. SYNCHRONIZATION ERRORS BETWEEN DRIVE SYSTEM (12) AND RESPONSE SYSTEM (13) WITH IMPULSE PERIOD $T=1$

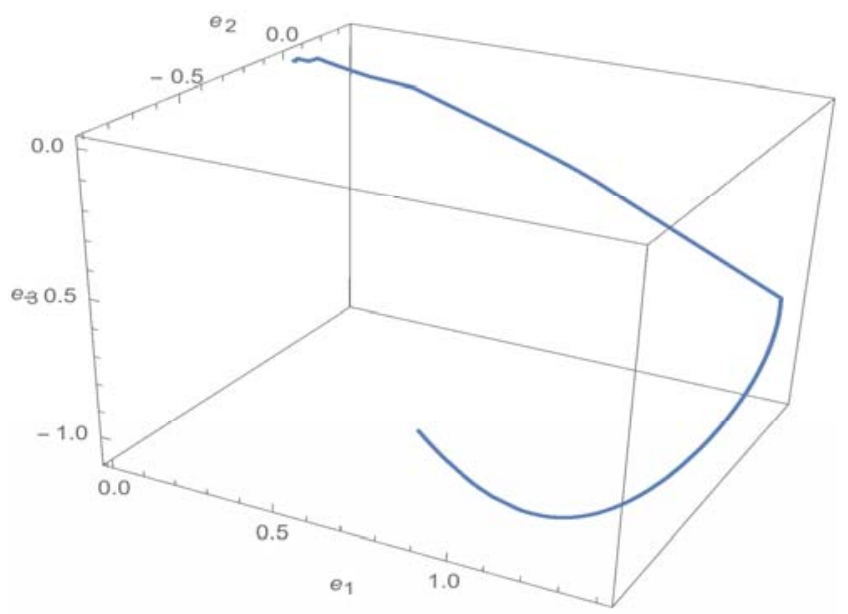

FIGURE VII. PHASE PORTRAIT OF SYNCHRONIZATION ERRORS BETWEEN DRIVE SYSTEM (12) AND RESPONSE SYSTEM (13) WITH IMPULSE PERIOD $T=1$ 


\section{CONCLUSIONS}

In this paper, the synchronization of the impulses neural network with a Gui chaotic strange attractor has been investigated based on the stability analysis of impulsive functional differential equation. Criteria for synchronization are derived. Some illustrative examples are finally included to visualize the effectiveness and feasibility of our main results.

\section{ACKNOWLEDGMENT}

This work was supported by the National Natural Science Foundation of Peoples Republic of China (Grant no. 60963025), the Natural Science Foundation of Hainan (Grant no. 613166).

\section{REFERENCES}

[1] Z. Chen, and J. Ruan, "Global stability analysis of impulsive cohencgrossberg neural networks with delay," Phys Lett A. 345(1): 101111, 2005.

[2] A. Cochocki, and R. Unbehauen. Neural networks for optimization and signal processing. John Wiley \& Sons, Inc., 1993.

[3] M. Cohen and S. Grossberg, "Absolute stability and global pattern formation and parallel memory storage by competitive neural networks," IEEE Trans Syst Man Cybernet. 13: 815-826, 1983.

[4] J. Hopfield, "Neural networks and physical systems with emergent collective computational abilities," Proc Nat Acad Sci USA. 79: 25542558, 1982.

[5] A. Samoilenko, N. Perestyuk, Y. Chapovsky, Impulsive differential equations. Singapore: World Scientific, 1995.

[6] R. Rakkiyappan, A. Chandrasekar, S. Lakshmanan, et al, "Exponential stability for markovian jumping stochastic BAM neural networks with mode-dependent probabilistic time-varying delays and impulse control," Complexity, 20(3): 39-65, 2015.

[7] Q. Song, and J. Zhang, "Global exponential stability of impulsive cohencgrossberg neural network with time-varying delays," Nonlinear Anal Real World Appl. 9(2): 500-510, 2008.

[8] Z. Chen, and J. Ruan, "Global dynamic analysis of general cohencgrossberg neural networks with impulse," Chaos, Solitons and Fractals. 32(5): 1830-1837, 2007.

[9] S. Arik. "Global asymptotic stability of a large class of neural networks with constant time delay". Phys Rev E. 311(6): 504-511, 2003.

[10] J. Cao, "Global stability analysis in delayed cellular neural networks," Phys Rev E. 59, 5940-5944, 1999.

[11] J. Cao, "New results concerning exponential stability and periodic solutions of delayed cellular neural networks," Phys Lett A. 307: 136147, 2003.

[12] Y. Li, "Global exponential stability of bam neural networks with delays and impulses," Chaos, Solitons and Fractals. 24: 279-285, 2005.

[13] K. Gopalsamy, and H. He, "Stability in asymmetric hopfield nets with transmission delay," Physics D. 76(4): 344-358, 1994.

[14] Z. Gui, and W. Ge, "Existence and uniqueness of periodic solutions of nonautonomous cellular neural networks with impulses," Physics Letters A. 354(1): 84-94, 2006.

[15] Z. Gui, and W. Ge, "Periodic solution and chaotic strange attractor for shunting inhibitory cellular neural networks with impulses," Chaos. 16(3): 033116, 2006.

[16] Z. Gui, and W. Ge, "Impulsive effect of continuous time neural networks under pure structural variations. International Journal of Bifurcationand," Chaos. 17(06): 2127-2139, 2007.

[17] Z. Gui, and W. Ge, "Periodic solution for nonautonomous bidirectional associative memory neural networks with impulses," Neurocomputing. 70: 2517-2527, 2007.

[18] Z. Gui, and W. Ge, "Existence and global exponential stability of periodic solutions of recurrent cellular neural networks with impulses and delays," Mathematics and Computers in Simulation. 9: 14C29, 2008.
[19] R. Amritkar, and N. Gupte, "Synchronization of chaotic orbits: the effect of a finite time steps,". Physical Review E. 47(6): 3889, 1993.

[20] T. Yang, and L. Chua, "Generalized synchronization of chaos via linear transformations," International Journal of Bifurcation and Chaos. 9(01): 215-219, 1999 .

[21] T. Yang, and L. Chua, "Impulsive stabilization for control and synchronization of chaotic systems: theory and application to secure communication," IEEE Transactions on Circuits and Systems I. 44(10): 976-988, 1997.

[22] T. Yang, and L. Chua, "Impulsive control and synchronization of nonlinear dynamical systems and application to secure communication," International Journal of Bifurcation and Chaos. 7(03): 645-664, 1999. 